\title{
Enhanced Facilitation of Biotechnology Education in Developing Nations via Virtual Labs: Analysis, Implementation and Case-studies
}

\author{
Shyam Diwakar*, Krishnashree Achuthan, Prema Nedungadi, and Bipin Nair, *Member, IEEE, IACSIT
}

\begin{abstract}
Methods for educating students in biotechnology require intensive training in laboratory procedures. Laboratory procedures cost Universities in terms of equipment and experienced guidance which often come short in many developing countries. Universities need revitalizing approach and well-adapted curriculum especially in terms of laboratory practice. For enhanced education at the level of University-level laboratory courses such as those in biology or biotechnology, one of the key elements is the need to allow the student to familiarize laboratory techniques in par with regular theory. The Sakshat Amrita virtual biotechnology lab project focusing on virtualizing wet-lab techniques and integrating the learning experience has added a new dimension to the regular teaching courses at the University. Establishing virtual labs requires both domain knowledge and virtualizing skills via programming, animation and device-based feedback. This paper reports a cost-effective process used in virtualizing real biotechnology labs for education at Universities. The major challenge in setting up an effective knowledge dissemination for laboratory courses was not only the scientific approach of biotechnology, but included the virtualization aspects such as usage/design scalability, deliverability efficiency, network connectivity issues, security and speed of adaptability to incorporate and update changes into existing experiments. This paper also discusses an issue-specific case-study of a functional virtual lab in biotechnology and its many issues and challenges.
\end{abstract}

Index Terms - virtual lab, e-learning, biotechnology virtual labs, virtual neurophysiology, sakshat amrita.

\section{INTRODUCTION}

Software technologies developed by academic institutions as well as industries worldwide is revolutionizing

Manuscript received December 20, 2009. This work is funded under the Sakshat project of National Mission on Education through ICT, Department of Higher Education, Ministry of Human Resource Department, Government of India.

S. Diwakar is with the Amrita School of Biotechnology, Amrita Vishwa Vidyapeetham (Amrita University), Amritapuri, Clappana P.O., Kollam, Kerala, 690525 India (Corresponding author, phone: 91-476-2803116; fax: 91-476-2899722; e-mail: shyam@ amrita.edu).

K. Achuthan is with School of Engineering, Amrita Vishwa Vidyapeetham (Amrita University), Ettimadai, Coimbatore, Tamil Nadu, 641105 India.

P. Nedungadi is with School of Engineering, Amrita Vishwa Vidyapeetham (Amrita University), Amritapuri, Clappana P.O., Kollam, Kerala, 690525 India.

B. Nair is the Dean of Amrita School of Biotechnology, Amrita Vishwa Vidyapeetham (Amrita University), Amritapuri, Clappana P.O., Kollam, Kerala, 690525 India. the educational system. In particular, the use of virtual reality techniques is emerging as a new possibility in imparting training to students. Simulation is the most effective tool in training students in the use of sophisticated as well as complicated instruments that are routinely employed in modern biological and chemical laboratories. This technology circumvents the use of expensive and hazardous biological and chemical agents which toxic to the experimentalists as well as to the environment. Above all, the virtual lab technology is cheap as well as cost effective.

Many universities and research institutes from developed nations have realized the potential of this concept and have already launched their own virtual laboratories on the web, which are accessible to people around the world. It has already been established that the Virtual Lab enables the students to understand the underlying principles and the theory behind laboratory experiments. The procedure for operating an analytical instrument can be simulated by a mathematical and/or empirical model. Using this virtual model, the student is trained in optimization, calibration and method development for the simulated sample. E-learning plays and will play an important role in diverse regions such as India where the traditional lab facilities at Universities are not very well localized to suit requirements of all sub-regions. With multi-campus scenarios as in some Universities including ours, offering cross-disciplinary courses needs to exploit the use of extensive e-learning facilities (unpublished observation but see [3]).

Biotechnology lab courses richly rely upon new up-to-date content and various techniques that require a new synergy of knowledge and experimental implementation. Hence a new kind of experimental science that can be brought as a virtual simulation based laboratory is necessary. The developments of the virtual labs include mathematical techniques in biology to study, to hypothesize and to demonstrate complex biological functions. However virtual labs in heavy engineering topics such as analyzing nanomaterials with high-power microscopes and lab courses in biotechnology or biology will also have to exploit multiple techniques besides simulators alone as many scenarios cannot be reproduced mathematically while retaining the "real" lab-like feel.

In this paper, we focus on the development on the virtualization of biotechnology lab courses through a combination of techniques to try completing the learning experience as that of a regular University laboratory. 


\section{Why ViRTUAL LABS?}

The main reasons to focus on creating virtual labs for University education are many [1]. One of the primary reasons include the cost and lack of sufficient skill-set for facing the current growth in biotechnology sector. The setup cost of laboratories puts a large overhead on the educators. The Universities also need to setup laboratories to educate sufficient target group with the details of common biotechnological techniques and protocols [5].

Another new motivation is the need to introduce and focus well-explored potential virtual lab areas which use computational methods, mathematical modeling and biophysics, computational biology and computational neuroscience. Computational biology and biophysics are upcoming areas and most techniques derive basis from real laboratory experiments. Another intention of using virtual labs via a computational approach is to train young scientists in the field of the mathematical thinking for life sciences and related environments. Main goals of cross-disciplinary sciences include the need to ensure that the students will be able to integrate different exhaustive models into a larger framework, i.e. in the perspective of comprehensive biological systems such as cells and biological networks. Such a role will also give an overview of the modeling approaches that are most appropriate to describe life-science processes. For the everyday biologist, the major use of virtual labs will also be in the learning perspective of advanced but common-to-use simulation tools.

Virtual labs and use of virtual tools should lead to an increase the awareness of a crucial need for standard model descriptions. Most simulators and common-use tools require various formats and schema and with the explosion of data, the use of virtual labs across the country or across multiple countries is also intended to unite educators to work towards common model descriptions and standardization of their data.

For the biotechnology sector, a highly favoring motivation for the shift to the virtual lab paradigm is the explosion of data-rich information sets, due to the genomics revolution, which are difficult to understand without the use of analytical tools. Also, recent development of mathematical tools such as chaos theory to help understand complex, nonlinear mechanisms in biology seems to push the need for information-rich virtual labs in simulation domain.

To aid further, an increase in computing power which enables calculations and simulations to be performed that were not previously possible, have set a new trend in the concept and use of computing. Simulations in the past that needed more intensive computers now can plainly be run through long battery-life laptops [2], given that in many cases laptops today even host servers.

A slightly different reason that also pushes the concept of virtual labs for undergraduate and master level education at the Universities also seems to be an increasing interest in in silico experimentation due to ethical considerations, risk, unreliability and other complications involved in human and animal research.

Given all above reasons and motivation, virtual labs are today's experimental approach towards a newer trend in future education. However the virtual lab environments are still under severe testing and newer models seems to switch to more intelligent and adaptive platforms that can yield an efficient knowledge dissipation. One such common model is the adaptive learning system (ALS) currently employed by many e-learning applications strewed on the internet.

\section{ADAPTIVE LEARNING}

To enhance education, there is a great need for individualized courseware to provide educational content that fits to the learner's learning style and current knowledge base. Effectiveness of adaptive learning supported by student's profiles $[7,8]$ has been and is being widely studied.

The schema of virtual labs was based on one of our studies. Amrita learning is an adaptive learning platform that attempts to emulate a one-on-one motivation of a teacher who estimates the student's knowledge level and learning speed, effectively responds to a student's needs and then provides feedback to the class teacher and the student. Intelligent class monitoring is used to identify the students who have learning records that are different from those of their peers [7]. Since University education faces a broader spectrum of disciplines and backgrounds, every student may be different from others in many ways. Academically, they could be progressing too fast, or too slow, or simply need different tutoring. Adaptive learning allows individual personalized attention to these learner scenarios.

AVIEW [3] and Amrita learning were the precursors and framework of reference for Sakshat Amrita Virtual Labs. Amrita Learning uses an adaptive assessment algorithm to determine a student's prior preferences, knowledge, skills and attitudes. Each topic presented to student was evaluated with a questionnaire-type quiz that served as an assessment tool.

The following were the key features for our adaptive learning system: 1) Supplement to class room teaching 2) Web based 3) Adaptive 4) Assessment 5) Adaptive Learning after completion of Assessment 6) Feedback mechanism for improvement 7) Content aligned to National Standards.

The technical architecture of the model system was modular with interface and logic clearly distinct (see [9]). It was a robust extension of the basic intelligent tutoring model where knowledge about the domain, teaching and student profile is tracked and used [17] Evaluation of Amrita Learning was based on deployment in 2 schools with 2275 students for a duration of 21 weeks and main tests comprised of lessons in mathematics.

An average survey of the VL framework software was performed and the tests are shown in Table 1. It is to be noted that for the Virtual Labs the average time spent on an experiment increased as with time (see Fig. 1) probably indicating the familiarization of the software favored extended use.

In order to test adaptive learning framework, we used the criteria similar to [10]. Feedback is usually a significant part of the assessment as students need to be informed about the results of their (current and/or overall) performance. 


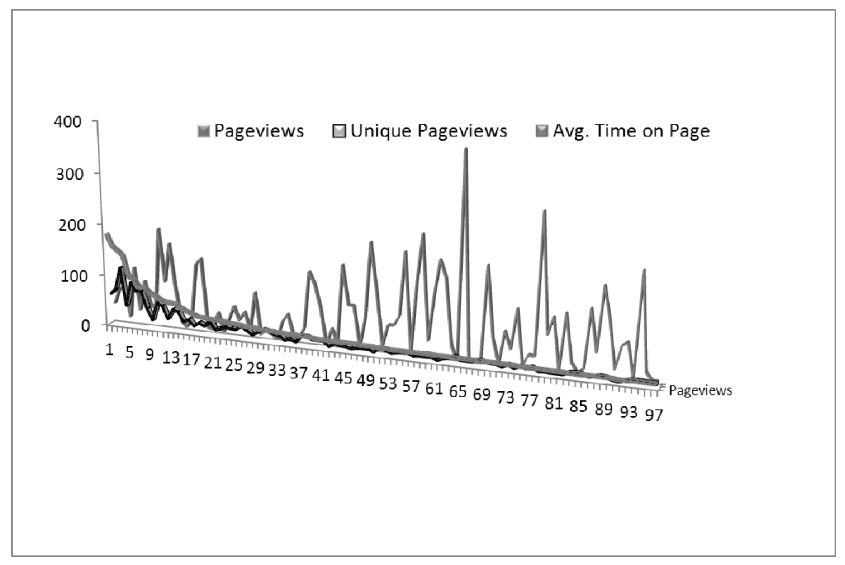

Fig. 1. Amrita Virtual Lab software web usage trend.

Feedback is usually not used as an evaluator but an assessment tool for student quality. With that in mind, the virtual lab evealuation criterion was focussed on measuring and estimating the student's involvement in the particular experiment of a particular lab. A way to increment the quantity and timing of feedback is to provide enough detail. Through animation, we have also increased evaluatory criterion and details in the virtual environments. It was noted that in more than 95 experiments performed by more than 30 people within a particular time-window there were more than 91\% of appreciation (further statistics pending, data not shown) when two experiments, one with detail oriented interactive animation and other without interaction were delivered to assess the involvement of the students in terms of their self-assessment.

\begin{tabular}{|c|c|c|c|}
\hline $\begin{array}{l}\text { VL framework } \\
\text { and Amrita } \\
\text { Learning: } \\
\text { Downtime } \\
\text { Study (6 } \\
\text { months) }\end{array}$ & Item & Issues & $\begin{array}{l}\text { Downtime } \\
\text { in last } 6 \\
\text { months }\end{array}$ \\
\hline \multirow[t]{5}{*}{$\begin{array}{l}\text { Server side } \\
\text { downtime }\end{array}$} & $\begin{array}{l}\text { Web server } \\
\text { downtime }\end{array}$ & $\begin{array}{l}\text { Problems with } \\
\text { apache web } \\
\text { server. Web } \\
\text { server crash, } \\
\text { server not } \\
\text { reachable from } \\
\text { some remote } \\
\text { locations etc }\end{array}$ & 45 minutes \\
\hline & $\begin{array}{l}\text { Database } \\
\text { downtime }\end{array}$ & $\begin{array}{l}\text { Problems with } \\
\text { database. } \\
\text { Database hang, } \\
\text { reboots etc }\end{array}$ & 0 minutes \\
\hline & $\begin{array}{l}\text { Performance } \\
\text { issues/Latency }\end{array}$ & $\begin{array}{l}\text { Performance } \\
\text { issue due to peak } \\
\text { usage, slow } \\
\text { queries, etc }\end{array}$ & \\
\hline & $\begin{array}{l}\text { Software } \\
\text { downtime }\end{array}$ & $\begin{array}{l}\text { For major } \\
\text { software updates, } \\
\text { debugging issues } \\
\text { etc }\end{array}$ & $\begin{array}{l}2 \text { hour in } 6 \\
\text { months } \\
\text { (scheduled } \\
\text { downtime } \\
\text { often at off } \\
\text { peak hours) }\end{array}$ \\
\hline & $\begin{array}{l}\text { Datacenter } \\
\text { downtime }\end{array}$ & $\begin{array}{l}\text { Downtime due to } \\
\text { power supply } \\
\text { issues at data } \\
\text { center. UPS }\end{array}$ & 30 minutes \\
\hline
\end{tabular}

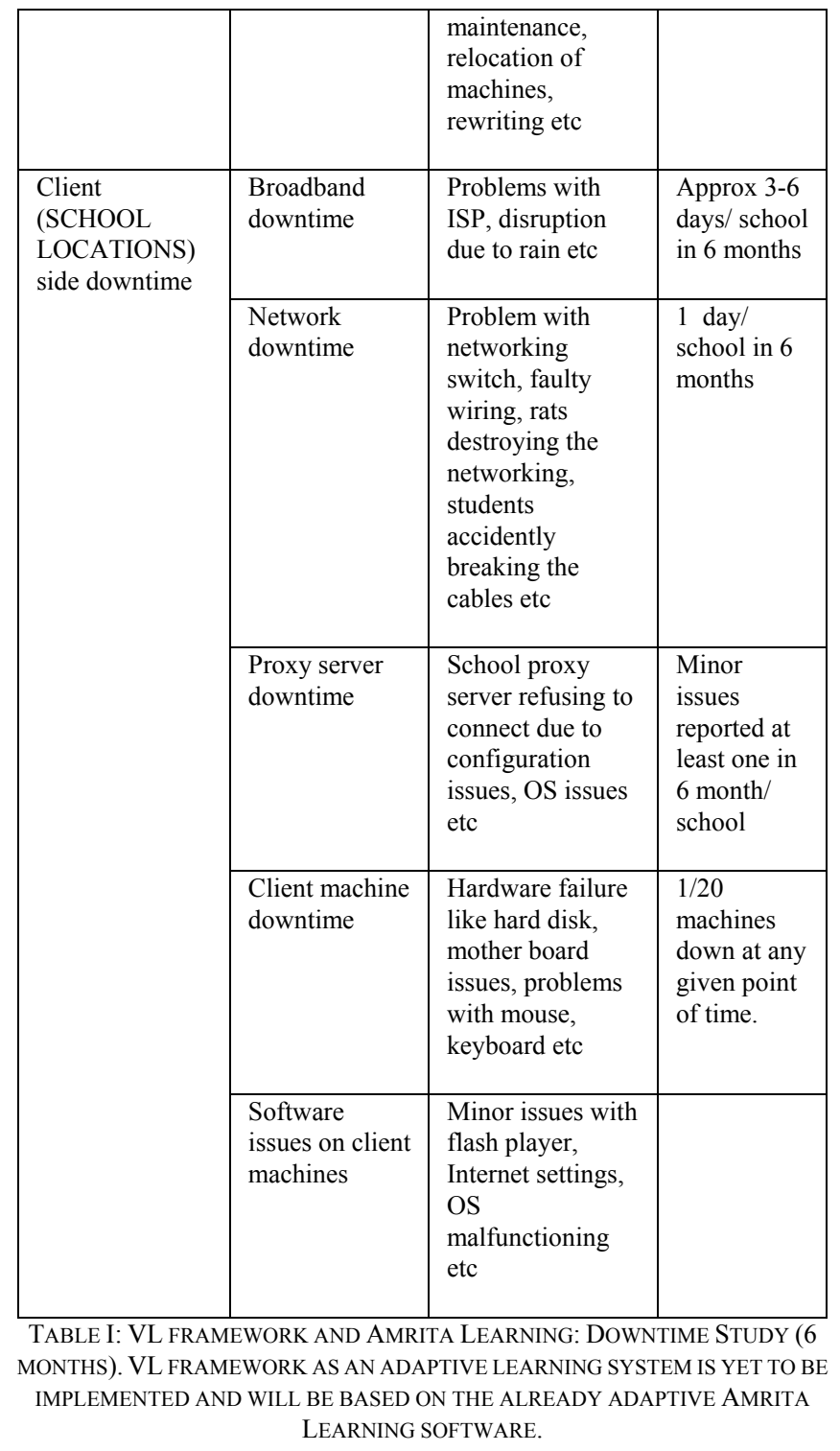

\section{TECHNIQUES - ANIMATION, MATHEMATICS AND VIRTUALIZATION}

The key learning component in many biological laboratories is the complexity of the procedure and details of the step-by-step protocol carried out in the laboratory. Although some of these biological processes can be replaced by mathematical equations modeling the system, most of the "feel" is in performing the detailed procedure which is not derived from sets of equations. Graphical animations deliver a high degree of the reality to the virtual labs through their seeming closeness to the appearance and feel of the lab. Graphical animations also cut out the complexity of the modeling process by increasing the "feel" of experiment. Like the proverb goes, "a picture is worth a thousand words", animations reveal better information that cannot be easily conveyed via text alone or static illustration.

In our biotechnology virtual labs, the animation type of experiments include the use of 2D flash based animations for illustrating detailed procedures such as wet lab protocols and heavy engineering techniques that are out of scope for simulation due to various reasons like complicated equations, numerical issues in simulation, lack of modeling data etc. Besides animation, another common technique in our virtual 
labs included engineering-based approaches such as remote-triggered experiments or remote-controlled experiments.

The very common and research-inspiring approach is the use of mathematical simulators to model biological and biotechnological processes or sub-processes. Although mathematics has long been intertwined with the biological sciences, an explosive synergy between biology and mathematics seems poised to enrich and extend both fields greatly in the coming decades. Among the various scenarios to study biology and disseminate information effectively and efficiently, includes the use of e-learning as a medium to offer courses.

Applying mathematics to biotechnology for virtual lab creation has recently turned into an explosion of interest in the field. The NASA virtual laboratory or the HHMI virtual labs at Howard Hughes Medical Institute or the Utah genetics virtual laboratory are some examples.

For our labs, a combination of user-interactive animation, mathematical simulations, remote-trigger of actual equipment and the use of augmented perception haptic devices are used to deploy effectively the real laboratory feel of a biotech lab online.

\section{MAJOR CHALlENGES}

Setting and developing AMRITA virtual labs (see Fig. 2) as a complete learning experience has not been an easy task. Amongst the major challenges we faced included usage/design scalability, deliverability efficiency, network connectivity issues, security and speed of adaptability to incorporate and update changes into existing experiments.

Owing to the scientific domain, biotechnology lends the following challenges to establishing virtual labs:

1) The development of analytical solutions in the arena is limited as biological processes are typically non-linear and are coupled systems of differential equations in various forms.

2) The mathematics behind models is hidden by their complexity and appears refined through simulation platforms.

3) Most simulation platforms need direct hands-on experience between teachers and students.

4) The number of students that can be catered at any given time is restricted.

5) Besides, such courses also need simultaneous theoretical explanations which may need classroom-like scenarios with video presentations, white-board and other tools. We could overcome the issue here using a collaborative suit, AVIEW [3].

6) There are not many courses in India developed for this scenario.

In order to address some of these issues and to overcome restrictions, we deployed virtual lab experiments webhosted simulators. The virtual lab was based on a website that was designed for favorable use within intranets and internets. However, efficiency depended on the internet bandwidth and connectivity. Our target was any campus with a download link of 256kbps should suffice. To retain this compatibility the animations had to be size-delimited. To overcome the problem, longer experiments had to be sliced to smaller portions, each loading in sequence. This was possible as we maintained the virtual lab experiments as flash animations (Adobe, USA). Having labs in flash environments allowed the scalability and access although flash based action script programming needed additional programmers and training.

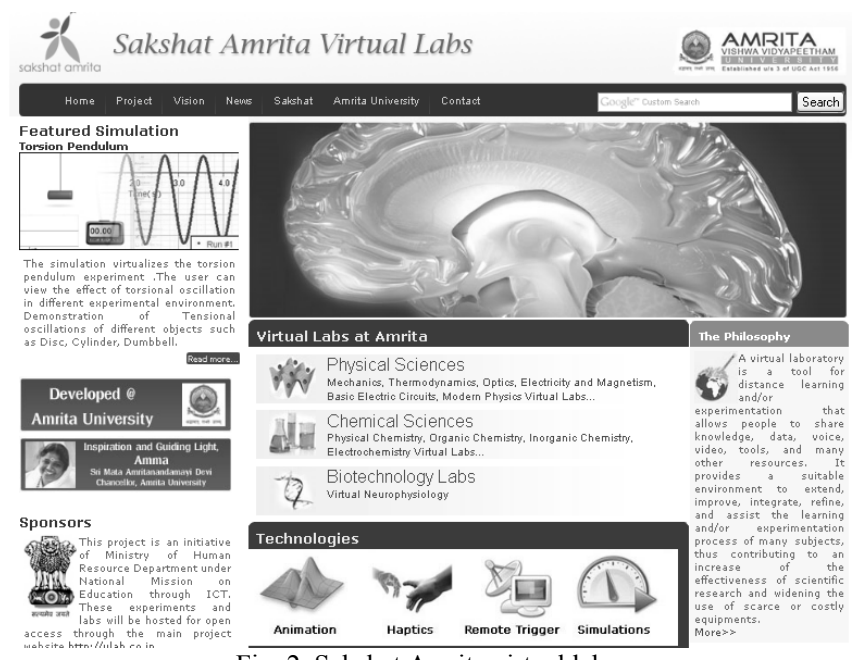

Fig. 2. Sakshat Amrita virtual labs.

Other e-learning issues such as student-teacher collaboration via chat, video interfacing etc. are overcome via AVIEW-like environment [3]. The intention of the virtual labs was also to extend the facility to develop an applied computational laboratory.

\section{Methodology}

Amongst others, the focus of having and designing virtual labs was also based on John Keller's ARCS model of motivation. Design of courses, simulations and models for computational approaches in biology will be the highlight. A lot of attention was on courses whose content will be applicable to the existing P.G. programs.

For all biotech virtual labs, we had set the following lab-level objectives as general guidelines.

1) Virtual labs should be adaptive. An adaptive e-learning system is a system in which modifies its behavior (the learning process) in response to the changes in the learners input data and information gathered from various teaching process. It should be able to incorporate data and user changes as and when possible.

2) Introduce and focus virtual lab areas in core computational and protocol-based biotechnological sciences.

3) To train young scientists in the field of the mathematical thinking for life sciences and related environments.

4) To ensure that they will be able to integrate different exhaustive models into a larger framework, in the perspective of a comprehensive biological systems such as cells and biological networks.

5) To give an overview of the modeling approaches most appropriate to describe life-science processes.

6) To give a practical introduction to advanced but 
common-use simulation tools.

7) To increase the awareness of a crucial need for standard model descriptions.

The implementation of animation and simulation based virtual labs was mainly done in Action Script 3 in Adobe flash in order to bring better definition to 2-D graphics. Action script allowed flash swf files as output thereby allowing both a better look-and-feel and an enhanced interactivity with the software. The physics simulator tools worked reasonably well. For one of the "more academic" simulators, we also used Sun Java based Graphic Java Toolkit to draw traces and plot graphs. We did not use java as a programming medium in our learning tool except in one simulator to make sure we have complete cross-OS, cross-browser compatibility, to reduce initial loading time and also to consider support for the commercial operating systems such as Microsoft's Windows platform that support flash better than Java plug-in.

We did not use the Amrita Learning Environment in its full functionality for the virtual labs. The minor intention was to deploy preliminary platform with a learning environment and later render the environment adaptive and intelligent as per the user-audience. The main reason to precursor with such a test was cost-efficiency. Cost-efficiency of e-learning programs has been increasingly important because some institutions have failed due to the lack of well-thought out financial plans (see $[11,12])$.

Virtual Labs use self-assessment based on questionnaire to evaluate user's experience. Although not implemented, an advanced form of the lab is being planned to include teacher's assessment, peer-assessment and collaborative assessment. Teacher assessment will actually have a "real" instructor on the deployment site to evaluate the lab user/student. Peer-assessment will include any student or teacher to assess another. Collaborative assessment will include both the instructor and the student to perform assessment on the completion of an experiment.

For our installation and deployment, we focused to reduce internet downtime. A 2004 study indicated that overall downtime costs companies an average of $3.6 \%$ of annual revenue (internet sources, see www.sentinelbussiness.it) indicating leading causes for downtime being software failure and human error. Through our studies, we managed to reduce unnecessary events and maintain downtime to less than 27 minutes for 6 months (not as in Amrita Learning software, see Table I). However, this could be because of our lack of full incorporation of the complex adaptive learning system as it was done for the schools where it was tested. However a test on real-time upgrade to such a model based on our previous experiences (data not shown) with Amrita learning [9] indicated that overall loss of virtual lab in terms of downtime will be significantly less.

\section{CASE Study: VIRTUAL NeURophysiology LABORATORY}

Amongst others, the focus of having and designing virtual labs was also based on John Keller's ARCS model of motivation. Design of courses, simulations and models for computational approaches in biology will be the highlight. A lot of attention was on courses whose content will be applicable to the existing P.G. programs.

Our preliminary studies in the biotech sector were on neurophysiology techniques. The virtual neurophysiology laboratory provides an opportunity for students to substitute classroom physiology course with detailed techniques and protocols of a real laboratory. Besides the material like chemicals, physiology demands extensive knowledge and experience from the instructor. For example, rat brain slicing protocol which is the first experiment (in the virtual lab) takes approximately 6-10 hours to complete training and about 2-3 weeks to train one student in a real laboratory.

With the focus on time [6] and learning know-how, we adapted the usual lab experimental protocols as user-interactive animations of the neurophysiology lab experience. The work involved both animators and programmers. For some experiments such as brain slice preparation, animations were sufficient whereas for some others such as Hodgkin-Huxley neuronal model ([4], see Fig. 3.) for demonstrating behavior of single neurons, we used Java based simulator. The same simulator was embedded into other experiments such as voltage clamp protocol and current clamp protocol to allow the student to see the corresponding behavior as seen in real neurons [16].

A new set of experiments being currently developed include the use of electronic resistance-capacitance(RC) circuits that could be remotely triggered as mimicking the electrical dynamics of a passive neuronal membrane. Passive neuronal membranes are modeled as RC-circuits with high resistance and low capacitance (for more details see [16]).

Also as part of the labs, we follow a particular formatting for each experiment within the lab. The goal was to allow the student to study the theory, the approach and do a self-test before actually going into the simulator or the virtual experiment. Covering some explanations and incorporating the same theory into the actual "lab" part of the experiment has been one of the primary goals. Each experiment in the labs (especially in Biotechnology) opens by default with the textual theory, which can also be randomly accessed by clicking on the icon "theory".

All the control and experimental parameters are explained in the "manual". The instructor and the student are informed on how various parameters change in the experiment in the very context of the virtual experimental lab procedure. For those experiments that have both an animation learning component and simulator component, each of the user controls and the variable parameters are explained. Also included in the manual is a help that actually explains the usage of radio controls and icons covered by the experiment. The intention was to evaluate the basic info that once the student completes the familiarization process by going through the theory and manual sections, he/she can take a "self-evaluatory" quiz module that chooses to test the student on some questions based on the theory background of the experiment.

The "simulator" tab actually leads to the experiment workbench. "Protocol for brain slicing" that is actually a detailed lab process that would take 6-10 weeks for 
post-master's student to learn and about 3-10 hours per procedure. That experiment we have virtualized by means of an interactive action script based animation. The second neurophysiology experiment concerns the modeling of a neuronal cell. In this case we have used a Flash based learning component along with a HH-simulator of a biophysical neuron.

The "assignment" icon is the lab experiment question with which intention the student performs the experiment. An instructor version of the assignment will include a model solved question or key tips in case of a protocol-like experiment. Additional reading material and reference information and other details will be found in our "misc info" icon.

Among the various methodologies the lab covers simulation-based, animation-based and remote-triggered experiments. The simulator was that of a bio-realistic model cell and was combined with an interactive animation-based learning-tool made using Flash. Maldarelli et al. [13] report the advantage of virtual lab demonstration as an effective lab tool. The remote-triggered experiments were based on real electronic circuits that mimic the phenomenon observed in neuronal cells. The basic behavior of Resistance-capacitance circuits that can be modified remotely by a user to study and imitate real neuronal circuits as he/she does in a neuronal biophysics laboratory on a patch of a neuronal membrane.

We also tested the virtual lab via a questionnaire-based feedback for overall quality. Among the major questions, several virtualizations related questions were presented in the questionnaire. The general developer/designer related questions included in the lab were to rate the experiment that was most recently completed, extent of control on the interface, closeness to lab environment and feel, measurement and analysis of data, user-manual quality, adequacy of bibliography and references, results interpretation, whether any clear information was gained by using the virtual lab, any problems faced, how helpful the lab was and overall motivation.

\section{COST OF VIRTUAL LABS}

In order to estimate the true financial cost of our virtual lab project, we had to include both project development and delivery and maintenance costs. As indicated by Kruse (see [14]), design of courseware needed more initial costs than instructor-led learning but delivery and maintenance is affordably cheaper. We estimate, based on Amrita learning software experience that there will be negligible costs for maintain web-based experiments. The main post-deployment costs included administration and maintenance. The administration and maintenance estimates included tracking of user-behavior, technical support, content updates and technology updates. Student material development, instructor costs and subject expert costs were included in the development expenses.

\section{Some Evaluatory Setbacks And Associated FEEDBACK}

What we know from the Virtual Lab studies performed is that user-involvement in assessment is vital for improving the knowledge-experience for the user. Self-assessment hints preliminary results but are not comprehensive. Users tend to show implicit behavior patterns indicating favor of the tool rather than the experiment for their choice of vote. Interactional voting behavior is also dependent on age and other characteristic learner attitudes. In our studies, younger students mostly at the undergraduate level evaluated the tool using mid-range scores compared to the varying yet favorably high votes of the Master's and Graduate students in the feedback assessment. Although this may need further testing, we believe that scores from the higher age-experience level indicated statistically relevant reliability much more than undergraduates (data not shown due to pending experiments).

Overall, 27 Master's level students who helped in intensive evaluation of the Virtual lab platform as part of their regular class-room course, appeared predominantly positive about the value of virtual labs in e-learning, but anxieties were also expressed about the potential for e-labs to replace face-to-face teaching and labs in the economically challenged regions of the country apart an indication either of the value on the personal and face-to-face tutoring through an expressed preference for it. Students who were positive about their experiences of virtual labs indicated that they had received appropriate introductions and felt supported by staff, indicating the importance of sound inductions into the use of institutional systems and technologies.

\section{CONClusion ANd Further REMARKS}

As a first comprehensive experience, we have dived into what is known as "virtual labs" and our approach methodology and issues were discussed above. The virtual lab protocols for neurophysiology and related sciences have been a successful complement to the usual theoretical education that happens at our school of biotechnology at the level of masters and undergraduate education. Although the elements can be improved, our approach to virtualization has answered many key results in establishing the virtual lab features such as teacher-independent/teacher-friendly approach to e-learning.

We tried to avoid the most usual failures in e-learning labs [15] by focusing to avoid the common failures. Our design issues were based on a successfully tested e-learning software environment [9] and included a clear identification and analysis of the real problem associated with University laboratory courses. Each virtual lab included overall strategic design decision such as structure of the courses, technologies employed and mode of experiment. Each experiment and the lab was considered with instructional design and elements that were evaluated so to motivate the learner experience. Such elements included the choice of graphical front-ends and authoring tools. We had also previously estimated issues related to dissemination for rapid, efficient and cost-effective usability taking into consideration both pedagogical and infrastructural complicacy. 
As next stage we plan to incorporate and embed other features which are now a part of Amrita learning software [9] and AVIEW [3] stand-alone collaborative suite to the virtual labs to enhance learning experience. Many features currently considered to be incorporated include selective student interaction by an instructor, instructor-instructor private collaboration when multiple teacher log on at the same time, video interface with selective response, attendance/number of hours logging, message posting, etc. Particular lab features that may enhance lab experience such as saving the state of an experiment, output lab reports in multiple formats, automated scoring and reporting are being considered for deployment. We are also planning to incorporate feedback as a feed-forward in the virtual lab adaptive learning tool.

The studies using adaptive learning software for a virtual lab combined animation, user-interaction, simulation and access to remote equipment has led to newer collaboration amongst academia to study and test new possibilities and reuse skill-set in cross-disciplinary sectors. A separate fall-out from this project was that the lab has helped to support vocational training for laboratory technicians who want to upgrade their positions. We hope to incorporate and test all features as and while we develop newer labs within the virtual lab framework. Some student/instructor behavior related studies for enhancing virtual lab environments is also being considered.

From our experience, the most vital requirement for each virtual lab is that of technical coordinators and subject matter experts whose inputs improve the lab's knowledge bank and usability. The Virtual lab project is already online for public preview via http://sakshat.amrita.ac.in and http://vlab.co.in.
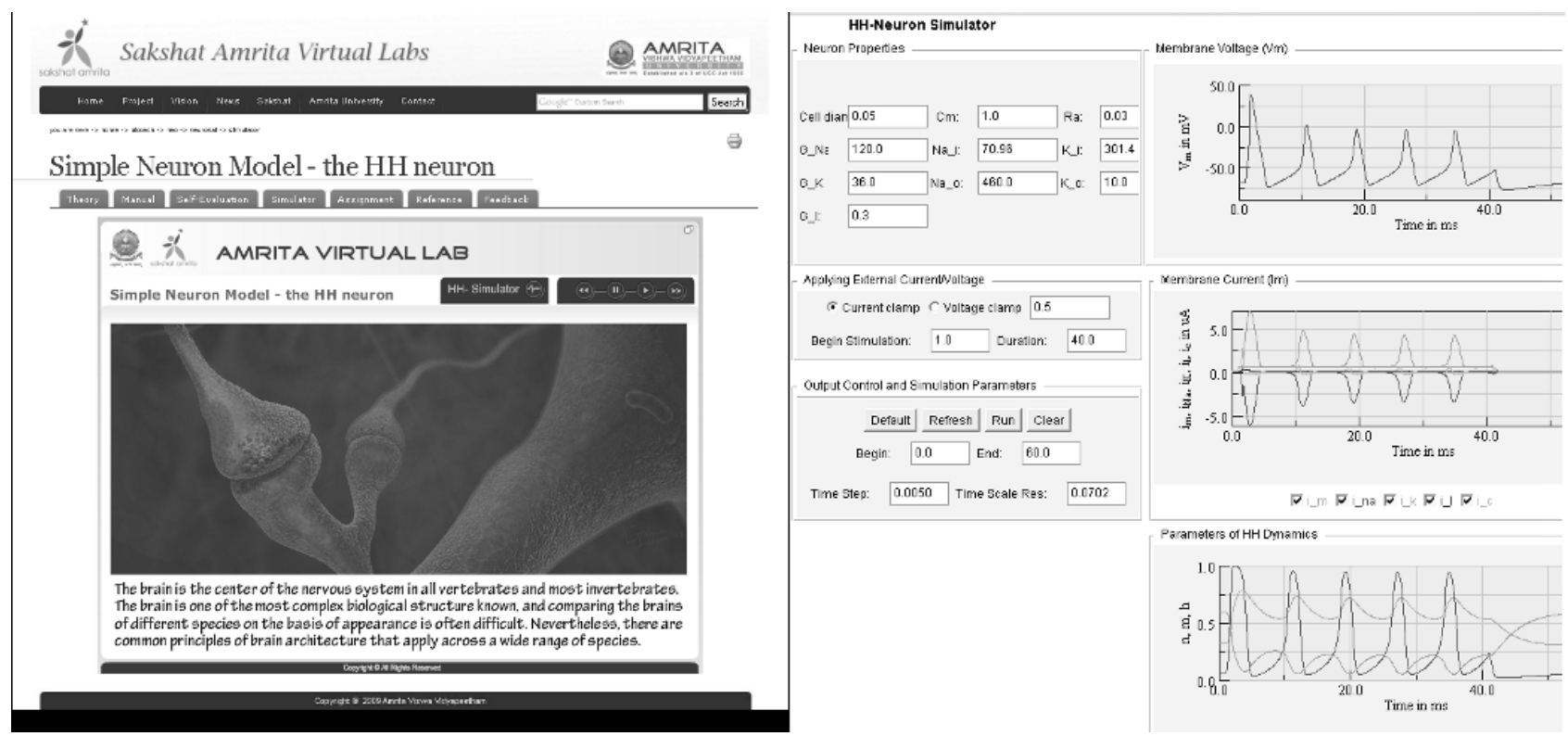

Fig. 3. A sample virtual neurophysiology lab experiment. Left side shows the lab experiment and right side plot shows the simulator and settings.

\section{ACKNOWLEDGMENT}

This project derives direction and ideas from the Chancellor of Amrita University, Sri Mata Amritanandamayi Devi. The authors would also like to acknowledge the contributions of Dr. Geeta Kumar, Mr. Kartik Iyer of the Amrita School of Biotechnology, Dr. Venkat Rangan, the Vice-Chancellor, Mr. Kamal Bijlani of Amrita E-learning TIFAC CORE, Ms. Bhavani Bijlani of Amrita University-Vocational training initiatives, Mr. Raghu Raman of Amrita University and Mr. Rathish of Amrita learning initiatives.

\section{REFERENCES}

[1] Auer M., Pester A., Ursutiu, D., Samoila C., Distributed virtual and remote labs in engineering, IEEE International Conference on Industrial Technology, Vol. 2, 2003, pp. 1208-1213.

[2] Aycock J., Crawford H., deGraaf R., Innovation and technology in computer science education (Proceedings of the 13th Annual Conference on Innovation and Technology in Computer Science Education, Madrid(Spain), 2008, pp. 142-147.

[3] Bijlani K., Manoj P., Rangan V., VIEW: A Framework for Interactive eLearning in a Virtual World, Proceedings of the Workshop on
E-Learning for Business Needs 2008/BIS, Innsbruck(Austria),2008, pp. 177-187.

[4] Hodgkin A.L., Huxley A.F., A quantitative description of membrane current and its application to conduction and excitation in nerve. Bull Math Biol, 1990, Vol 52, pp. 25-71.

[5] O'donoghue J., Singh G., Dorward L., Virtual education in universities: A technological imperative, British Journal of Educational Psychology, 32(5), 2001, pp.511-523.

[6] Rohrig C., Jochheim A., The Virtual Lab for controlling real experiments via Internet, Proceedings of the 1999 IEEE International Symposium on Computer Aided Control System Design, 1999, pp. 279 -284 .

[7] Brusilovsky P. Adaptive and Intelligent Technologies for Web-based Education; Special Issue on Intelligent Systems and Teleteaching, Künstliche Intelligenz, 1999.

[8] Brusilovsky P. Methods and Techniques of Adaptive Hypermedia. User Modeling and User-Adapted Interaction, Kluwer academic publishers Vol. 6, pp. 87-129, 1996.

[9] Nedungadi P and Raman R. Effectiveness of Adaptive Learning with Interactive Animations and Simulations. Proceddings of the 2nd International Conference on Computer Engineering and Applications (ICCEA 2010), Bali, Indonesia, March 2010.

[10] Katterfeld C and Konig G. Analysis of e-learning software and guidelines for quality assurance in photogrammetry, remote sensing and gis. Proceedings of the ISPRS Congress, Beijing, 2008.

[11] Wentling t and Park J. Cost Analysis of E-learning: A Case Study of A University Program, Proceedings of the AHRD, University of Illinois at Urbana-Champaign, p.1-11, 2002. 
[12] Morgan BM. Is distance learning worth it? Helping to determine the costs of online courses. Eric number 446611, 2000.

[13] Maldarelli GA, Hartmann EM, Cummings PJ, Horner RD, Obom KM, Shingles R and Pearlman RS. Journal of microbiology \& biology education, pp. 51-57, May 2009.

[14] Kruse K. Measuring the Total Cost of e-Learning, http://www.e-learningguru.com/articles/art5_2.htm.

[15] Romiszowski A. How's the E-learning Baby? Factors Leading to Success or Failure of an Educational Technology Innovation Educational Technology, 44(1), Jan-Feb 5-27, 2004.

[16] Koch, C. Biophysics of Computation: Information Processing in Single Neurons,Stryker, M. (ed.) Oxford University Press, 1999.

[17] Wenger E. Artificial Intelligence and Tutoring Systems: Computational and Cognitive approaches to the communication of knowledge. Morgan Kaufman Ed., 1987. 